\title{
Ultrasensitive Detection of Amines by a Trace Amine-Associated Receptor
}

\author{
Jingji Zhang, ${ }^{1 \star}$ Rodrigo Pacifico, ${ }^{1 \star}$ Dillon Cawley, ${ }^{1}$ Paul Feinstein, ${ }^{2}$ and Thomas Bozza ${ }^{1}$ \\ ${ }^{1}$ Department of Neurobiology, Northwestern University, Evanston, Illinois 60208, and ${ }^{2}$ Department of Biological Sciences, Hunter College, City University \\ of New York, New York, New York 10065
}

The mammalian main olfactory pathway detects volatile chemicals using two families of G-protein-coupled receptors: a large repertoire of canonical odorant receptors and a much smaller set of trace amine-associated receptors (TAARs). The TAARs are evolutionarily conserved in vertebrates, including humans, suggesting an indispensible role in olfaction. However, little is known about the functional properties of TAARs when expressed in native olfactory sensory neurons. Here we describe experiments using gene targeting, electrophysiology, and optical imaging to study the response properties of TAAR-expressing sensory neurons and their associated glomeruli in mice. We show that olfactory sensory neurons that express a subset of the TAAR repertoire are preferentially responsive to amines. In addition, neurons expressing specific TAARs, TAAR3 or TAAR4, are highly sensitive and are also broadly tuned-responding to structurally diverse amines. Surprisingly, we find that TAAR4 is exquisitely sensitive, with apparent affinities for a preferred ligand, phenylethylamine, rivaling those seen with mammalian pheromone receptors. We provide evidence that this unprecedented sensitivity is mediated via receptor coupling to the canonical odorant transduction cascade. The data suggest that the TAARs are evolutionarily retained in the olfactory receptor repertoire to mediate high-sensitivity detection of a biologically relevant class of odorous stimuli.

\section{Introduction}

To detect structurally diverse volatile chemicals, the mammalian olfactory system has developed a large repertoire of chemosensory receptor genes. There are two known families of seventransmembrane receptors expressed by olfactory sensory neurons (OSNs) in the main olfactory epithelium of the mouse: $>1000$ canonical odorant receptors (ORs) and a much smaller family of trace amine-associated receptors (TAARs) (Liberles and Buck, 2006; Nei et al., 2008). Although both ORs and TAARs are G-protein-coupled receptors, the TAARs are more closely related to biogenic amine receptors (Lindemann et al., 2005; Liberles and Buck, 2006) and are evolutionarily conserved in vertebrates, including humans (Lindemann et al., 2005; Hashiguchi and Nishida, 2007; Hussain et al., 2009), suggesting that they serve a critical chemosensory function.

In the mouse, 14 of the 15 intact TAAR genes are expressed in the olfactory system (Liberles and Buck, 2006). Ligands have been identified for six mouse TAARs using heterologous expression,

\footnotetext{
Received Sept. 8, 2012; revised Nov. 19, 2012; accepted Dec. 13, 2012

Author contributions: R.P., P.F., and T.B. designed research; J.Z., R.P., D.C., and T.B. performed research; J.Z., R.P., and T.B. analyzed data; R.P. and T.B. wrote the paper.

*J.Z. and R.P. contributed equally to this work.

This work was supported by National Institutes of Health/National Institute on Deafness and Other Communication Disorders Grant 1R01DC009640 (T.B.), the Whitehall Foundation (T.B.), and Brain Research Foundation (T.B.), and National Institutes of Health/National Institute of General Medical Science Grant 5SC1GM088114 (P.F.). We thank Adam Dewan and Matt Wachowiak for comments on the manuscript and the Gene Targeting and Transgenic Resource Centers at Rockefeller University for assistance with gene targeting.

The authors declare no competing financial interests.

Correspondence should be addressed to Thomas Bozza, Department of Neurobiology, Northwestern University, 2205 Tech Drive, Hogan 2-160, Evanston, IL 60208. E-mail: bozza@northwestern.edu.

DOI:10.1523/JNEUROSCI.4299-12.2013

Copyright $\odot 2013$ the authors $\quad 0270-6474 / 13 / 333228-12 \$ 15.00 / 0$
}

and these receptors have been shown to be narrowly tuned to amines (Liberles and Buck, 2006; Ferrero et al., 2011; Ferrero et al., 2012). It has been suggested that identified TAAR ligands may serve as social cues and/or predator-derived chemicals (kairomones) in rodents. In particular, trimethylamine is enriched in the urine of sexually mature male mice and activates TAAR5 (Liberles and Buck, 2006). $\beta$-Phenylethylamine is enriched in carnivore urine, elicits aversive responses in rodents, and activates TAAR4 (Ferrero et al., 2011). The data suggest that the TAARs may be specialized to detect chemical signals that carry specific information or that have a negative valence.

Recently, it was shown that TAAR-expressing OSNs form a distinct projection to glomeruli in the dorsal aspect of the main olfactory bulb (Johnson et al., 2012; Pacifico et al., 2012). More specifically, 10 of the 14 TAAR genes are selectively expressed by a subset of OSNs and are mapped to clustered glomeruli that respond selectively to low concentrations of amines (Pacifico et al., 2012). However, there are currently no data examining the odorant response properties of OSNs that express identified TAAR genes in any species. Consequently, little is known about the functional specificity of TAARs in vivo or whether TAAR ligands identified in vitro activate their corresponding TAARexpressing OSNs. In addition, the transduction cascade that mediates TAAR responses in their native neurons is unknown.

We used gene targeting, electrophysiology, and in vivo imaging to characterize the functional properties of TAAR-expressing OSNs and their corresponding glomeruli in mice. Our data show that TAAR-expressing OSNs are selectively (although not exclusively) responsive to amines. OSNs expressing TAAR3 and TAAR4 and their corresponding glomeruli are more broadly tuned than expected, and TAAR4 is extraordinarily sensitive, ex- 
hibiting robust responses to subpicomolar odorant concentrations, comparable with mammalian pheromone receptors (Leinders-Zufall et al., 2000). We provide evidence that the TAAR responses are likely mediated by coupling to the canonical odorant transduction cascade. Together, our data argue that the TAARs, and TAAR4 in particular, serve as high-affinity amine detectors in mammalian olfactory systems.

\section{Materials and Methods}

All procedures were approved by the Northwestern University Institutional Animal Care and Use Committee.

Gene targeting. Generation of the Venus $\rightarrow$ Taar4, Taar4-IREStauCherry and Taar3-IRES-tauVenus targeted strains was described previously (Pacifico et al., 2012). To generate the human TAAR5 (hTAAR5) swap ( $h$ Taar $5 \rightarrow$ Taar4-IRES-tauVenus), the coding sequence of $h$ Taar 5 was amplified from a bacterial artificial chromosome (BAC) clone (RP11-295F4), flanked with AscI restriction sites, and preceded by a Kozak consensus sequence. The TAAR4 targeting vector was modified so that the native coding sequence was replaced with AscI and PacI sites. The hT5 coding sequence was inserted into the AscI site, followed by a PacI cassette containing the self-excising neo gene ACNf (Bunting et al., 1999), an internal ribosome entry site, and the coding sequence for tauVenus, a fusion between the microtubule-associated protein tau and Venus yellow fluorescent protein (YFP) (Nagai et al., 2002). The targeting vector was linearized with XhoI and electroporated into E14 ES cells using standard methods. G418-resistant clones were screened by Southern blot using probes external to the targeting vector. Chimeras were generated by blastocyst injection into C57BL/6 embryos.

Electrophysiological recordings. Patch-clamp recordings were made from the dendritic knobs of semi-intact OSNs in epithelial explants (Ma et al., 1999). Male and female mice ( $n=52$; P7-P20) were anesthetized with ketamine-xylazine (200 and $15 \mathrm{mg} / \mathrm{kg}$, i.p., respectively), and the olfactory epithelium was removed and kept in oxygenated bath solution ( $95 \% \mathrm{O}_{2}-5 \% \mathrm{CO}_{2}$ ) containing the following (in $\mathrm{mm}$ ): $124 \mathrm{NaCl}, 3 \mathrm{KCl}$, $1.3 \mathrm{MgSO}_{4}, 2 \mathrm{CaCl}_{2}, 26 \mathrm{NaHCO}_{3}, 1.25 \mathrm{NaHPO}_{4}$, and 15 glucose, pH 7.4 (305 mOsm). The epithelium was transferred to a recording chamber and kept under continuous flow (1-2 $\mathrm{ml} / \mathrm{min}$ ) of oxygenated bath solution. All experiments were performed at room temperature.

The knobs of OSNs were visualized with an upright epifluorescence/infrared differential interference contrast (DIC) microscope equipped with a cooled CCD camera (SensiCam QE; Cooke Corporation) and a $40 \times$ water-immersion objective. Dendritic knobs of labeled OSNs were identified under fluorescence illumination and targeted under DIC. Patch pipettes were pulled from borosilicate glass with a P-97 horizontal puller (Sutter Instruments) and fire-polished using a microforge (MF 83; Narishige). Electrophysiological recordings were made using an EPC-10 amplifier controlled with Pulse software (HEKA). Perforated patch clamp was performed by including $260 \mu \mathrm{M}$ amphotericin $\mathrm{B}$ in the recording pipette, which was filled with the following solution (in $\mathrm{mm}$ ): $70 \mathrm{KCl}, 53 \mathrm{KOH}, 30$ methanesulfonic acid, 5 EGTA, 10 HEPES, and 70 sucrose, pH 7.2 with $\mathrm{KOH}$ ( $310 \mathrm{mOsm})$. The electrodes had tip resistances ranging from 8 to 10 $\mathrm{M} \Omega$ when filled with internal solution. The liquid junction potentials were corrected in all experiments. Signals were acquired at $10 \mathrm{kHz}$ and low-pass filtered at $2.9 \mathrm{kHz}$.

Odorants were applied with a pressure ejection system (PDES-02D; NPI Electronics) via a multi-barrel pipette placed $20 \mu \mathrm{m}$ downstream of the dendrite. Stimuli were delivered using a pulse length of $200 \mathrm{~ms}$. No responses were seen to pressure ejection of saline vehicle in any cells. Stimulus volume was measured by ejecting saline into mineral oil and measuring the diameter of the resulting bubble using a calibrated eyepiece micrometer. The average ejected volume for a $200 \mathrm{~ms}$ pulse was $5.8 \pm 1.3 \mathrm{nl}(n=20$ observations). Amine odorants were dissolved directly in bath solution or were solubilized in dimethylsulfoxide for some experiments. Identical results were obtained under both conditions. Stock odorant solutions were stored at $-20^{\circ} \mathrm{C}$ and diluted by adding bath solution. Odorant mixtures contained the following odorants: amines ( $\beta$-phenylethylamine, trimethylamine, $N$-methyl piperidine, ca- daverine, cyclohexylamine, propylamine); aldehydes (butyraldehyde, benzaldehyde, heptaldehyde, octyl aldehyde); acids (isovaleric acid, propionic acid, butyric acid, hexanoic acid); or ketones (isovalerophenone, acetophenone, butyrophenone, propiophenone).

Analysis and curve fitting were performed using Igor Pro 4.08J (Wave Metrics). Dose-response curves were fitted by the Hill equation: $I=$ $I_{\max } /\left(1+\left(\mathrm{EC}_{50} / C\right)^{n}\right)$, where $I$ represents the peak response, $I_{\max }$ the maximum current induced by the saturating concentration, $\mathrm{EC}_{50}$ the concentration at which the half-maximum response was reached, $C$ the concentration of odorant, and $n$ the Hill coefficient. Statistical tests were performed using Statview. The $t$ test was used for measuring the significance of difference between two distributions. All data are shown as mean \pm SEM.

Histology. Whole-mount images of olfactory epithelia and bulbs were performed using confocal microscopy on fresh, unfixed tissue. Combined immunohistochemistry/in situ hybridization was performed as described previously (Ishii et al., 2004; Bozza et al., 2009) using homozygous T4-ChR-YFP mice (Pacifico et al., 2012). In short, P21P30 mice were anesthetized and fixed through cardiac perfusion with ice-cold heparinized saline and $4 \%$ paraformaldehyde. Nasal tissue was dissected and postfixed at $4^{\circ} \mathrm{C}$ overnight, followed by $0.5 \mathrm{M}$ EDTA decalcification and $30 \%$ sucrose cryoprotection (both $4^{\circ} \mathrm{C}$ overnight). OCTembedded epithelium was frozen and sectioned at $12 \mu \mathrm{m}$. YFP was detected using a rabbit polyclonal antibody to Aequorea victoria green fluorescent protein (GFP) (Clontech). Riboprobes to signal transduction components were generated via in vitro transcription from PCRamplified templates with an incorporated T7 RNA polymerase promoter. Gene fragments were amplified from BAC DNA or from an olfactory epithelial cDNA library. Probe sequences are available on request. All imaging was performed using a Carl Zeiss LSM5 confocal microscope and analyzed with Carl Zeiss AIM software.

In vivo imaging. Glomerular imaging was done as described previously (Pacifico et al., 2012) with some modifications. Male and female mice were 8-12 weeks old and were heterozygous for both the OMP-spH (Bozza et al., 2004) as well as T4-RFP and/or T3-YFP alleles (Pacifico et al., 2012). Mice were anesthetized with urethane ( $1 \mathrm{~g} / \mathrm{kg}$, i.p.; Sigma) and chlorprothixene hydrochloride $(10 \mathrm{mg} / \mathrm{kg})$, followed by administration of atropine sulfate $(2.7 \mathrm{mg} / \mathrm{kg}$; Med-Pharmex). Mice were freely breathing throughout the experiment. For some experiments, imaging was done from one side while occluding the contralateral naris.

Odorants were applied using a custom-made, flow dilution olfactometer and controller (LASOM; RPMetrix) that was designed to avoid even minute levels of cross-contamination among the amines, which could be easily detected by the most sensitive TAAR glomeruli. To obtain low enough concentrations, amines were diluted in water and subsequently by flow dilution. Data were acquired using a NeuroCCD-SM256 camera and Neuroplex software (RedShirtImaging). Blank trials were subtracted from odor trials before analysis to compensate for photobleaching. Response maps were obtained by subtracting a $3.2 \mathrm{~s}$ temporal average preceding the stimulus from a $3.2 \mathrm{~s}$ temporal average encompassing the response peak. Responses are expressed as $\Delta F$ to account for the fact that the background synaptopHluorin $(\mathrm{spH})$ fluorescence is not correlated with the pool of indicator that reports neuronal activity (Bozza et al., 2004; McGann et al., 2005). For recordings from identified glomeruli, one glomerulus was chosen per mouse because multiple glomeruli within the same mouse are not independent. Stimuli were presented at least twice in a given experiment. Data shown are averages of two or more trials or the first trial in cases in which significant adaptation of the amine response was observed. Images were processed and analyzed in Neuroplex (RedShirtImaging) and NIH Image J software. Vapor concentrations were estimated using published vapor pressures (Estimation Programs Interface Suite, version 4.0; Environmental Protection Agency).

\section{Results}

TAAR-expressing OSNs are selectively responsive to amines

To examine the response profiles of TAAR-expressing OSNs, we recorded from genetically identified OSNs in gene-targeted mice. Odorant-evoked currents were measured by targeted patch- 
clamp recordings in voltage-clamp mode from fluorescently labeled dendrites in an epithelial explant preparation (Ma et al., 1999; Grosmaitre et al., 2006).

To gain an overview of the odorant specificity of OSNs expressing a variety of TAARs, we took advantage of a gene-targeted mouse strain $(\triangle T 4-Y F P)$ in which the coding sequence for the Taar4 gene is replaced with that of a fluorescent marker, Venus YFP. We showed previously that TAAR-expressing OSNs exhibit biased gene choice and are restricted to choose from among the TAAR gene repertoire (Pacifico et al., 2012). More precisely, OSNs that choose the T4 locus frequently choose from nine other dorsally expressed TAAR genes. Thus, in heterozygous mice, the population of OSNs expressing the $\Delta \mathrm{T} 4-\mathrm{YFP}$ allele ( $\Delta \mathrm{T} 4-\mathrm{OSN}$ ) coexpress 10 of the 14 TAARs (including the intact T4 allele in trans), giving rise to a labeled, diversified population of OSNs (Fig. 1A,B). By sampling this population, we could thereby functionally characterize sensory neurons that express a variety of TAAR genes. To determine whether TAAR-expressing OSNs are selectively activated by amines, we tested $\Delta \mathrm{T} 4-\mathrm{OSN}$ s with four odorant mixtures, each comprising acids, aldehydes, ketones, or amines (Fig. $1 B, C$ ). A high stimulus concentration was used to uncover even low-sensitivity responses. As a positive control, OSNs were also tested with the phosphodiesterase inhibitor isobutylmethylxanthine (IBMX), which is commonly used to activate the odorant transduction cascade (Firestein et al., 1991).

The vast majority of $\Delta \mathrm{T} 4-\mathrm{OSNs}$ ( 44 of 46 OSNs) exhibited responses to the amine mixture. Half of the cells responded exclusively to this mixture, whereas the other half responded preferentially to the amine mixture and exhibited smaller responses to non-amine mixtures (Fig. $1 C, D$ ). For comparison, we tested the mixtures on OSNs expressing class I or class II deletion alleles ( $\Delta \mathrm{S} 50-\mathrm{OSN}$ and $\Delta \mathrm{M} 72-\mathrm{OSNs}$ ) that preferentially express class I or class II ORs, respectively (Bozza et al., 2009). Neither population showed the same selectivity for the amine mixture (Fig. $1 E)$. We note that the amine mixture frequently elicited largeamplitude responses in $\Delta \mathrm{T} 4-\mathrm{OSN}$ - much larger than what is typically seen with canonical ORs at similar concentrations in this assay (our unpublished data). This observation indicated to us that at least some of the TAARs might be very sensitive to amines. Our mixture data indicate that a sampling of OSNs that express a majority of the TAARs are preferentially responsive to amines but can also exhibit small responses to non-amines when stimulated at high concentrations.

\section{TAAR-expressing OSNs are broadly tuned to amines}

Next, we examined the response profiles of OSNs expressing specific TAARs. To do this, we used mice in which OSNs expressing defined TAAR genes are labeled with fluorescent markers and targeted these cells for patch-clamp recording. To examine the response properties of OSNs expressing TAAR3 (T3-OSNs), we used the Taar3-IREStauVenus strain (Pacifico et al., 2012) in which OSNs expressing the endogenous Taar3 gene are labeled with a YFP (Fig. $2 A-C)$. T3-OSNs were tested with a set of six amines: $N$-methylpiperidine, cyclohexylamine, $\beta$-phenylethylamine, trimethylamine, isopentylamine, and cadaverine. Of these, T3 has been reported to respond specifically to isopentylamine and cyclohexylamine (Liberles and Buck, 2006). Unexpectedly, T3-OSNs responded to all six of the amines when stimulated at $1 \mu \mathrm{M}(n=9$ cells; Fig. $2 D, E)$. The effective stimuli included primary and tertiary amines and a diamine. At $1 \mu \mathrm{M}$, the most effective stimuli were isopentylamine and cyclohexylamine, with the latter eliciting the larger responses. The average response amplitude was 107.3 \pm 25.7 $\mathrm{pA}$ for isopentylamine and $157.6 \pm 31.1 \mathrm{pA}$ for cyclohexylamine
( $n=9$ cells). At $10 \mathrm{nM}$, T3-OSNs responded more specifically to isopentylamine $(75.3 \pm 8.2 \mathrm{pA})$ and cyclohexylamine $(47.7 \pm 7.7$ pA; $n=7$ cells; Fig. $2 E$ ). Because of limitations in space clamp, OSNs often exhibited action potentials riding on top of the recorded odorevoked currents (Fig. 2D). Interestingly, even small-amplitude current responses elicited increases in action potential firing rate, indicating that responses to all of these amines are likely transmitted to the bulb. Thus, T3-OSNs are more broadly tuned than would be predicted based on previous in vitro expression data (Liberles and Buck, 2006).

Next, we examined the response profiles of OSNs expressing TAAR4 (T4-OSNs) using the same set of amines. To do this, we used the Taar4-IRES-tauCherry strain in which OSNs expressing the endogenous Taar4 (T4) gene are labeled with a red fluorescent protein (RFP) (Fig. 3A-C). T4 has been reported to respond exclusively to $\beta$-phenylethylamine and structurally similar compounds in vitro (Liberles and Buck, 2006; Ferrero et al., 2011). Like T3, we found that T4-OSNs were broadly tuned, responding to the previously identified ligand $\beta$-phenylethylamine, but also to other structurally diverse amines (Fig. $3 D, E$ ). In addition, we observed that T4-OSNs were strikingly sensitive, exhibiting broad tuning and large responses at $10 \mathrm{~nm}(n=13$ cells $)$. At a lower concentration $(0.5$ $\mathrm{nM}), \beta$-phenylethylamine was clearly the preferred stimulus. The average amplitude was $112.1 \pm 19.3 \mathrm{pA}$ for $\beta$-phenylethylamine and $8-22 \mathrm{pA}$ for the other amines $(n=9$ cells $)$. At higher concentrations $(1 \mu \mathrm{M}), \mathrm{T} 4-\mathrm{OSN}$ s exhibited very-large-amplitude responses to all the tested amines (Fig. 4C), much larger than what is observed for T3 (Fig. 2E). Thus, our recordings show that both T3 and T4 are differentially sensitive but also broadly tuned to amines.

\section{TAAR4 expression is sufficient to account for the broad tuning of TAAR4-OSNs}

One possible explanation for the pronounced breadth of tuning is that TAAR-expressing OSNs could express multiple receptors. To determine whether all of the responses we observed in T4OSNs are attributable to the expressed T4 receptor, we genetically swapped the TAAR4 coding sequence to see whether this would eliminate responses to all of the effective stimuli. A similar approach has been used to test for specificity of endogenously expressed class II ORs (Bozza et al., 2002).

We generated a strain of mice in which the T4 coding sequence is replaced with that of a different receptor, human TAAR5 ( $h T 5 \rightarrow$ T4-IRES-tauVenus). In these mice, OSNs that would normally express the T4 receptor are forced to express hT5 protein instead, and the neurons are labeled with the fluorescent marker tauYFP (Fig. 4A, B). OSNs expressing the modified allele (hT5-OSNs) are located throughout the dorsal olfactory epithelium, in a pattern that is similar to that observed in T4-RFP mice (compare Figs. $3 \mathrm{~B}, 4 \mathrm{~B}$ ). In addition, axons of OSNs expressing hT5 coalesce into glomeruli in the dorsal aspect of the main olfactory bulb (Fig. 4B), in the same region as axons expressing T4 and other TAARs (Pacifico et al., 2012). This indicates that hT5 functions properly to mediate axon guidance when expressed in mice.

Swapping T4 for hT5 caused a dramatic alteration in odorant response profile. T4-OSNs responded robustly to all of the tested amines when tested at $1 \mu \mathrm{M}(n=4$ cells; Fig. $4 C)$. In contrast, hT5-OSNs exhibited only small responses to some of the amines (trimethylamine, cyclohexylamine, and $N$-methylpiperidine) at $1 \mu \mathrm{M}(n=4$ cells $)$. No responses were seen at concentrations below $1 \mu \mathrm{M}$ (data not shown). These results indicate that the robust responses to all of the amines that we observe in T4-OSNs are mediated solely by the expressed $\mathrm{T} 4$ protein. 
A

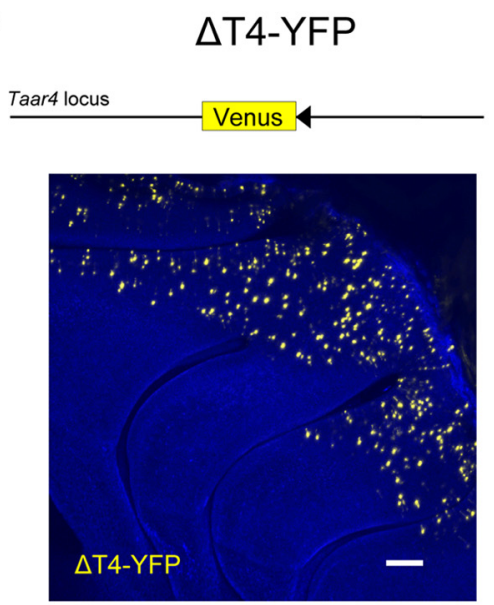

B

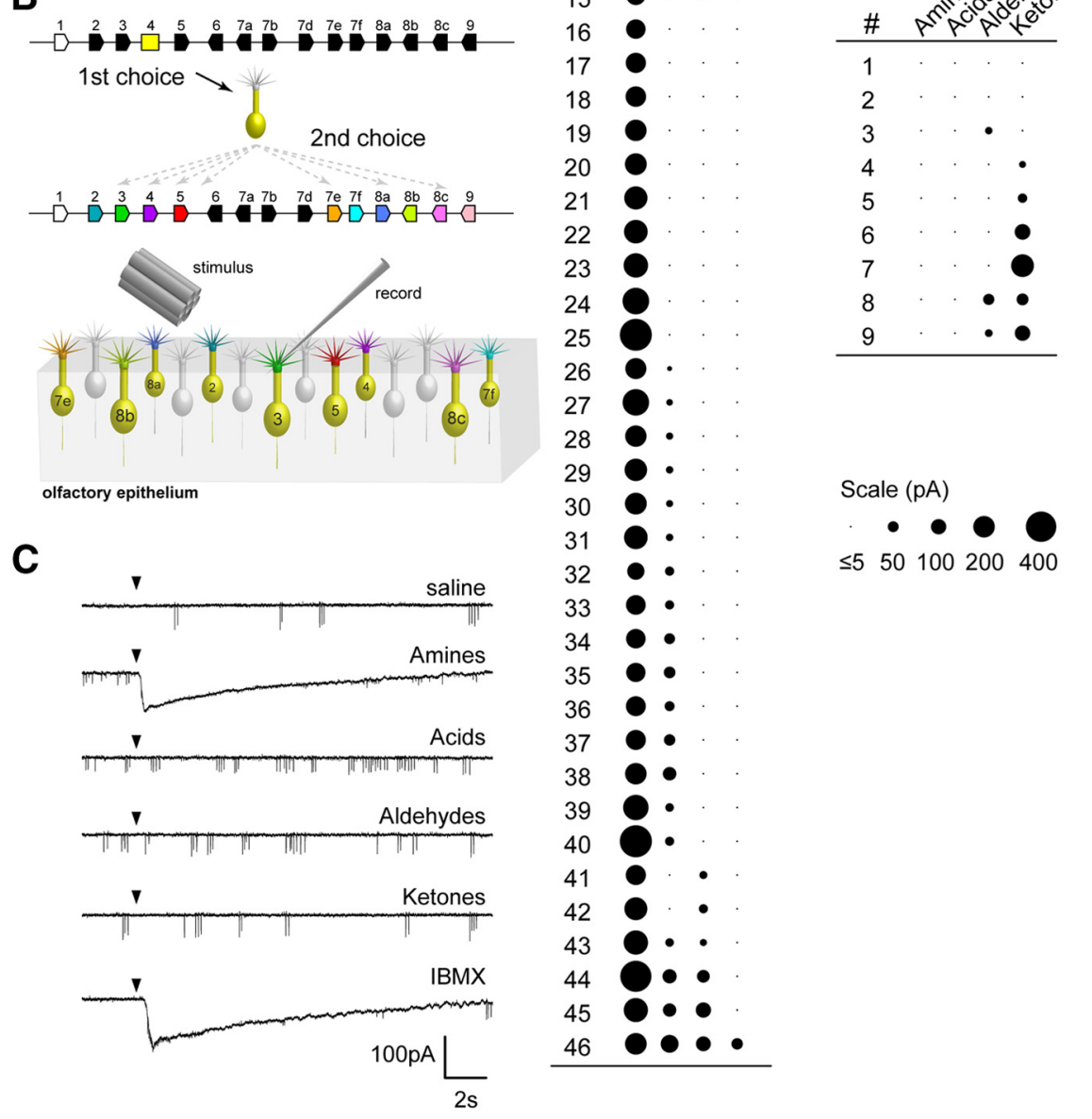

Figure 1. TAAR-expressing OSNs respond selectively to amines. $A$, Diagram of the T4 deletion in which the coding sequence for TAAR4 is replaced with that of Venus YFP (yellow), followed by a loxP site (black triangle). Confocal image of the olfactory turbinates in a $\Delta T 4-Y F P$ homozygous mouse showing a dorsal distribution of labeled cells. Scale bar, $200 \mu \mathrm{m}$. $\boldsymbol{B}$, Schematic of alternate gene choice in $\Delta T 4-Y F P$ OSNs. An OSN that chooses to express the $\Delta T 4-Y F P$ allele (yellow box) does not express a functional OR (yellow OSN with gray cilia) and goes on to make a second choice that is restricted to a subset of TAAR genes. Diagram of the TAAR cluster with the potentially chosen genes shown in color. Non-olfactory Taar 1 is shown in white. Non-chosen TAARs are shown in black. As a population, YFP-labeled OSNs coexpress various TAARs (yellow OSN with colored cilia). Fluorescent cells are targeted for patch recording. C, Odor-evoked currents in a $\triangle T 4-0 S N$ stimulated with 200 ms pulses of odorant mixtures containing amines, carboxylic acids, aldehydes, or ketones. High concentrations ( $1 \mu \mathrm{m}$ each odorant) were used to reveal the full breadth of tuning. Cell shown responded with robust inward currents (downward deflections) to the amines mix, to the positive control phosphodiesterase inhibitor IBMX, but not to other mixtures or to saline. For mixture components, see Materials and Methods. Action potential transients can be seen riding on top of the current traces. $\boldsymbol{D}$, Dot plots showing the response profiles across odorant mixtures for 46 IBMX-responsive $\triangle T$ T4-OSNs. Each row represents a single OSN. The area of the dot is proportional to
No ligands have been reported previously for human olfactory TAARs. To identify ligands, we screened 40 individual amines on hT5-OSNs. Of these, hT5OSNs responded best to $N, N$ dimethylethylamine, a structural analog of trimethylamine (Fig. 4D). When tested across concentration, the observed $\mathrm{EC}_{50}$ for $\mathrm{N}, \mathrm{N}$-dimethylethylamine was $1.2 \pm$ $0.6 \mu \mathrm{M}$ with a threshold of $10^{-7} \mathrm{M}(n=6$ cells). Our anatomical and physiological data together indicate that hTAARs can mediate both chemoreceptive and axon guidance functions when expressed in the mouse olfactory system.

TAAR4 is highly sensitive to amines

The large-amplitude responses observed in T4-OSNs suggested that T4 might be highly sensitive to amines. To examine this, we stimulated T4-OSNs with amines at multiple concentrations (Fig. 5A). In fact, T4OSNs were strikingly sensitive. The observed $\mathrm{EC}_{50}$ for $\beta$-phenylethylamine was $1.0 \pm 0.6 \times 10^{-12} \mathrm{M}$, with response thresholds near $1 \times 10^{-14} \mathrm{M}(n=8$ cells; Fig. $5 B)$. Even the amines that were not reported previously as T4 ligands in cell lines elicited responses at low concentrations (Fig. $5 B$ ). The mean $\mathrm{EC}_{50}$ values for cyclohexylamine and $N$-methylpiperidine were $7.7 \pm 4.4 \times 10^{-10}$ $\mathrm{M}(n=4$ cells $)$ and $5.1 \pm 1.7 \times 10^{-10} \mathrm{M}(n=$ 4 cells), with thresholds near $10^{-11} \mathrm{M}$. These responses are six orders of magnitude lower than those observed in cell lines and five to six orders of magnitude lower than what is observed for OSNs expressing canonical ORs (Grosmaitre et al., 2006, 2009; Zhang et al., 2012).

Next, we asked whether OSNs expressing TAARs other than T4 respond to amines at comparably low concentrations. To do this, we examined the sensitivity of T3-OSNs using its preferred ligands isopentylamine and cyclohexylamine. T3-OSNs were very sensitive to these ligands and responded over a broad concentration range (Fig. $5 C$ ). The mean $\mathrm{EC}_{50}$ for isopentylamine was $1.5 \pm 0.8 \times$ $10^{-8} \mathrm{M}$ with a threshold of $10^{-12} \mathrm{M}(n=4$ cells). Similarly, the mean $\mathrm{EC}_{50}$ for cyclohexylamine was $2.7 \pm 1.0 \times 10^{-7} \mathrm{M}$ with a threshold of $10^{-9} \mathrm{M}(n=5$ cells $)$. The mean $\mathrm{EC}_{50}$ for cyclohexylamine was significantly lower for T4 than for T3 ( $t$ test, $p<0.05)$. Thus, T3-OSNs are highly sen-

$\longleftarrow$

the peak response amplitude. $\boldsymbol{E}$, Dot plots showing the response profiles of IBMX-responsive OSNs from $\Delta S 50(n=10$ cells) and $\Delta M 72$ ( $n=9$ cells) mice that express class I and class II ORs, respectively. 
A

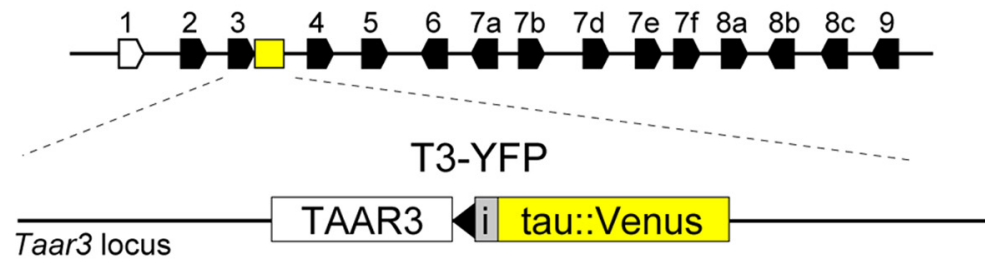

B

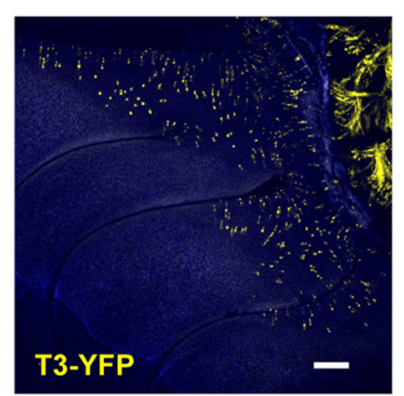

D
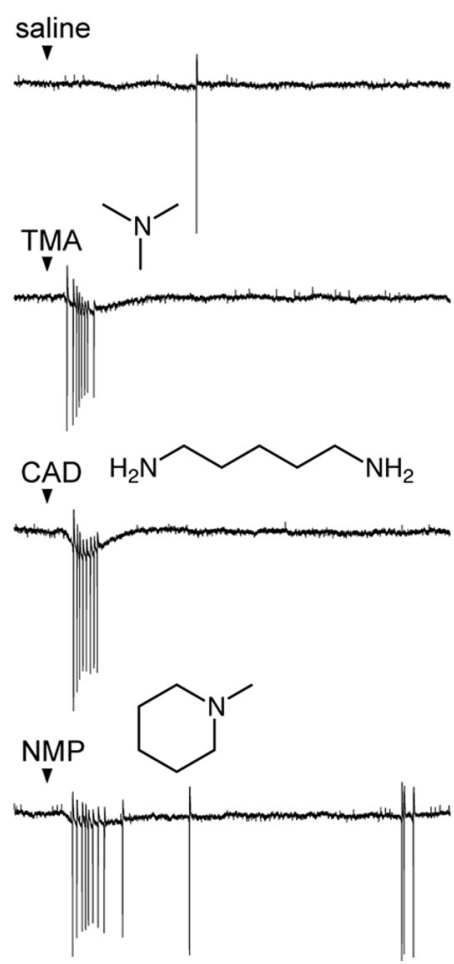

E

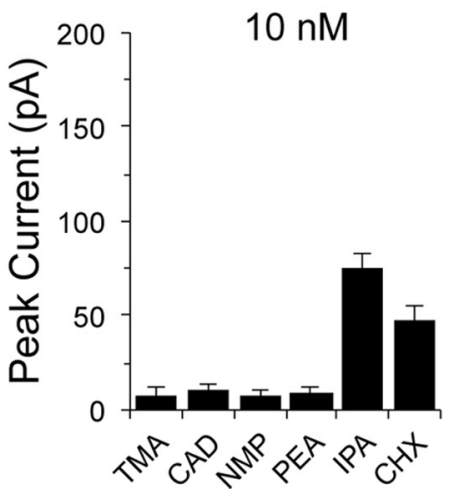

C
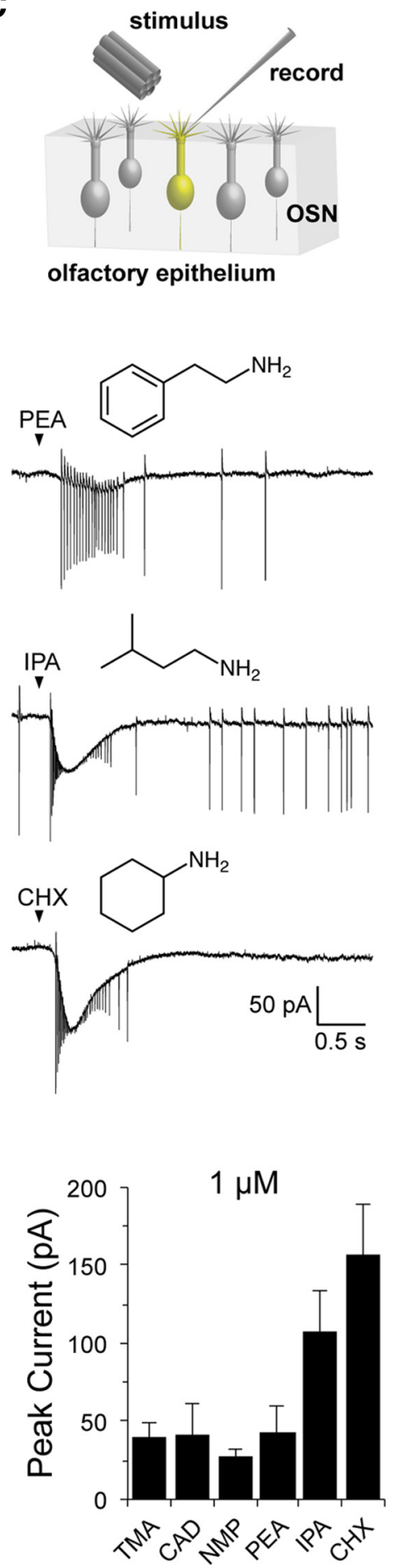

Figure 2. TAAR3-expressing OSNs are broadly tuned to amines. $\boldsymbol{A}$, Diagram of the TAAR gene cluster and the TAAR3 targeted allele. Polygons indicate gene orientation; olfactory TAARs are shown in black, and Taar1 is shown in white. The modified Taar3 locus contains an internal ribosome entry site (gray box marked " $\mathrm{i}$ ") and a fluorescent marker tauVenus (yellow box). Black triangle

sitive to amines but less sensitive than T4OSNs to known agonists.

To further test the sensitivity of OSNs expressing TAARs other than T4, we recorded from labeled OSNs in homozygous $\Delta \mathrm{T} 4-\mathrm{YFP}$ mice. In these animals, $\Delta \mathrm{T} 4-\mathrm{OSN}$ s coexpress a random sample of the dorsally expressed TAAR genes (Pacifico et al., 2012) except for T4, which is absent from the genome (Fig. 1A). To assess sensitivity among neurons that have different response profiles, we used a mixture of amines (trimethylamine, cadaverine, $N$-methylpiperidine, $\beta$-phenylethylamine, isopentylamine, and cyclohexylamine) at multiple concentrations. Five of 14 homozygous $\Delta \mathrm{T} 4-\mathrm{OSN}$ s responded to the amine mixture at a concentration below $1 \mu \mathrm{M}$ (Fig. 5D). Two exhibited thresholds in the picomolar range and $\mathrm{EC}_{50}$ values $\sim 2 \mathrm{~nm}$. However, we did not observe cells that were as sensitive as T4-OSNs in this sample. Thus, of the TAARs that we sampled, T4 appears to be the most sensitive.

TAAR4-expressing OSNs use the canonical odorant transduction cascade Given that TAAR-expressing OSNs comprise a genetically distinct cell population in the olfactory epithelium (Pacifico et al., 2012) and that T4-OSNs exhibit unusually high sensitivity, we tested whether TAAR-OSNs might signal through a distinct transduction pathway from typical ORs. ORs couple to an olfactory-specific G-protein $\left(\mathrm{G} \alpha_{\text {olf }}\right)$ that generates increases in cAMP via an olfactory-enriched adenylyl cyclase (type III). Increases in cAMP open cyclic nucleotide-gated channels (CNGA2/CNGB1b), which in turn pass $\mathrm{Ca}^{2+}$ ions that open calcium-gated $\mathrm{Cl}^{-}$ channels, most likely anoctamin 2 (Kleene, 2008; Stephan et al., 2009). To test for expression of olfactory signal transduction components, we performed

$\leftarrow$

indicates the location of a loxP site. $\boldsymbol{B}$, Confocal image of the olfactory turbinates in a T3-YFP heterozygous mouse showing labeled OSNs in the dorsal epithelium. Scale bar, $200 \mu \mathrm{m}$. C, Diagram of the electrophysiological recording configuration. Odor-evoked currents are recorded via perforated patch from the dendritic knobs of OSNs in a semi-intact preparation of the olfactory epithelium while odorants are puffed onto the cilia. $\boldsymbol{D}, 0$ dor-evoked currents measured in voltage-clamp mode in a genetically identified T3-YFP OSN. Odorants were presented for $200 \mathrm{~ms}$ at $1 \mu \mathrm{m}(n=9$ cells). T3-OSNs responded with robust inward currents (downward deflections) to odorants but not to saline. Action potential transients can be seen riding on top of the current recordings. $\boldsymbol{E}$, Response profiles of T3-YFP OSNs at $10 \mathrm{~nm}(n=7$ cells) and $1 \mu \mathrm{m}$ ( $n=9$ cells). Odors are trimethylamine (TMA), cadaverine (CAD), $N$-methylpiperidine (NMP), $\beta$-phenylethylamine (PEA), isopentylamine (IPA), and cyclohexylamine (CHX). 
A

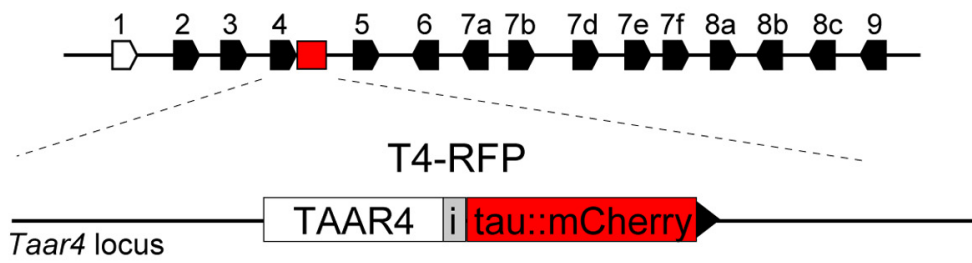

B

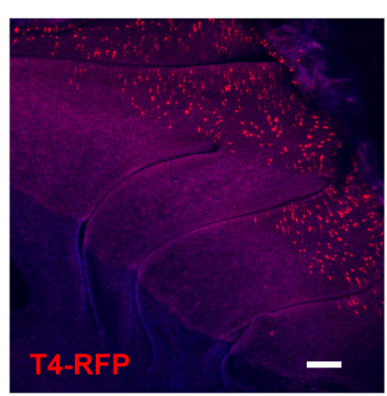

\section{C}

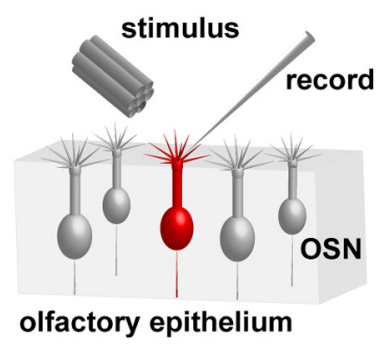

D

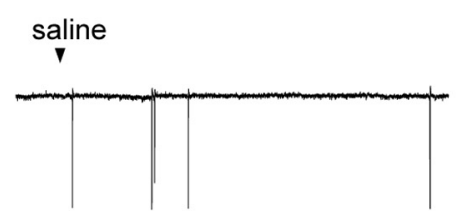

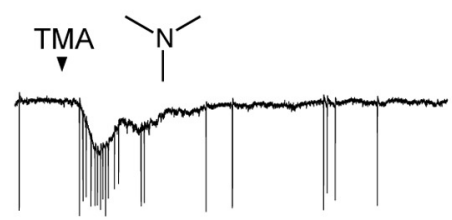
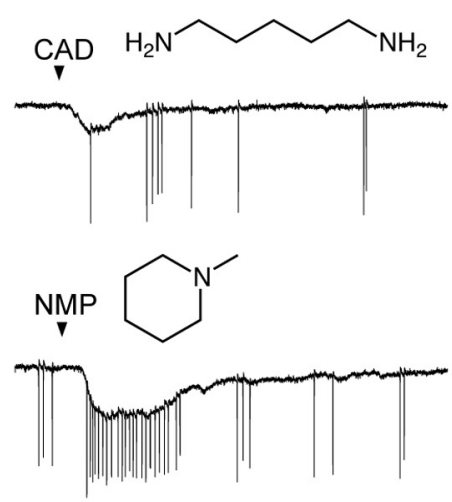

E
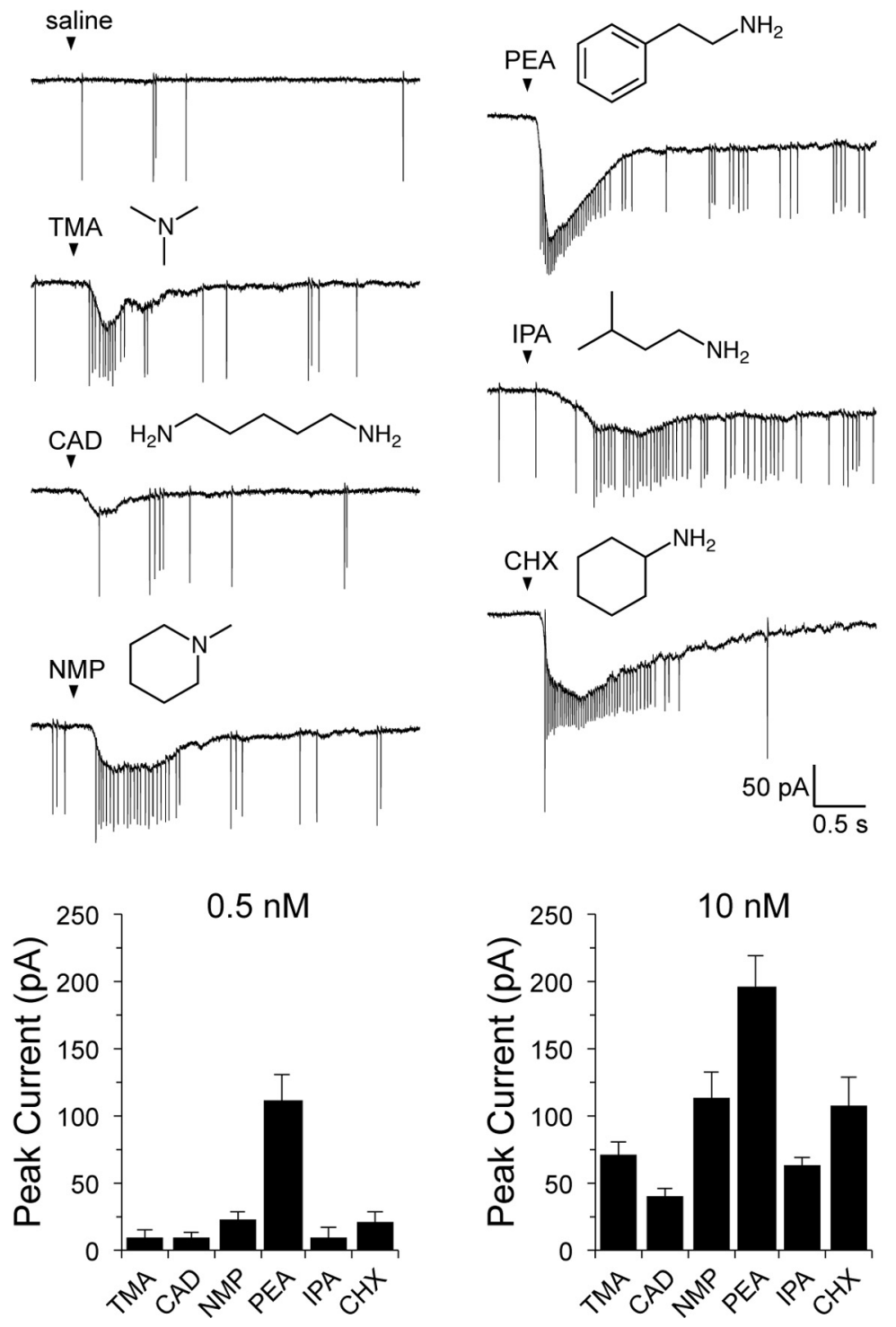

Figure 3. TAAR4-expressing OSNs are broadly tuned and highly sensitive. $\boldsymbol{A}$, Diagram of the TAAR gene cluster and the TAAR4 targeted allele. Annotations are as described in Figure 2. B, Confocal image of the olfactory turbinates in a T4-RFP homozygous mouse showing labeled OSNs in the dorsal epithelium. Scale bar, $200 \mu \mathrm{m}$. C, Odor-evoked currents are recorded from fluorescently labeled OSNs via perforated patch from the dendritic knobs. D, Odor-evoked currents in a genetically identified T4 -RFP OSN combined fluorescence in situ hybridization and immunohistochemistry on T4expressing OSNs. We find that T4-OSNs express $\mathrm{G} \alpha_{\text {olf }}$, consistent with previous findings (Liberles and Buck, 2006). In addition, T4-OSNs express other members of the canonical odorant transduction cascade, including ACIII, CNGA2, CNGB1b, PDE1c, PDE4a, Ric8b, and Ano2 (Fig. 6A).

If TAAR-expressing OSNs use the canonical odorant transduction cascade, known modulators of this pathway should induce or block transduction current in T4-OSNs. In fact, we observe that amine responses in $\mathrm{T} 4-\mathrm{OSN}$ s are blocked by the adenylyl cyclase blocker MDL12,330A (Fig. 6B). This block is similar to what is observed for OSNs expressing a canonical class II OR, M72 (Fig. 6B). These data are also consistent with the observation that TAAR-expressing OSNs respond to the phosphodiesterase inhibitor IBMX, which increases intracellular cAMP (Fig. 1C and data not shown). Thus, the highly sensitive amine responses in TAARexpressing OSNs are most likely mediated by coupling to the canonical odorant transduction cascade.

TAAR4 glomeruli are broadly tuned and highly sensitive to amines

Next, we examined whether the response properties observed for T3 and T4 in our in vitro electrophysiological assay are also observed in vivo when odorants are presented under more physiological conditions in the vapor phase. To do this, we imaged odor-evoked activity from genetically identified T3 and T4 glomeruli in anesthetized mice (Fig. 7). This was done using heterozygous $O M P-s p H$ mice in which the activity reporter $\mathrm{spH}$ is expressed in all mature OSNs (Bozza et al., 2004). Mice were also heterozygous for the T4-RFP allele, the T3-YFP allele, or both. Using appropriate fluorescence filters, we could separate the green, yellow, and red fluorescence signals of $\mathrm{spH}$, YFP, and RFP, respectively (see Materials and Methods), allowing us to identify T3 and T4 glomeruli in the same mice (Fig. 7C).

\section{$\leftarrow$}

Odorants were presented for $200 \mathrm{~ms}$ at $10 \mathrm{~nm}$. T4-OSNs responded with robust inward currents (downward deflections) to odorants but not to saline. Spontaneous and odor-evoked firing can be seen as transients riding on top of the current traces. $\boldsymbol{E}$, Response profiles of T4-RFP OSNs at $0.5 \mathrm{~nm}(n=9$ cells) and $10 \mathrm{~nm}$ ( $n=13$ cells). 0 dors are trimethylamine (TMA), cadaverine (CAD), N-methylpiperidine (NMP), $\beta$-phenylethylamine (PEA), isopentylamine (IPA), and cyclohexylamine (CHX). 
As we showed previously, the amines preferentially activated glomeruli in the dorsal caudal olfactory bulb (Pacifico et al., 2012), including our genetically tagged and neighboring glomeruli.

Consistent with our patch recordings, T4 glomeruli were preferentially responsive to $\beta$-phenylethylamine at low concentrations but were also broadly tuned at higher concentrations. In particular, isopentylamine, cyclohexylamine, and cadaverine elicited robust responses at nanomolar concentrations (Fig. 7A). $\beta$-Phenylethylamine was by far the most potent activator of T4 glomeruli. When the amines were presented at a fixed concentration (2 nM), T4 glomeruli exhibited much larger responses to $\beta$-phenylethylamine than to any of the other amines (ANOVA, $p<0.001$; Fig. 7D).

T4 glomeruli were also highly sensitive to amines. The lowest concentration at which we obtained reliable responses to $\beta$-phenylethylamine was $100 \mathrm{pm}$ (Fig. $7 B, E)$, lower than the most sensitive glomeruli typically observed for canonical ORs (Oka et al., 2006). In fact, T4 glomeruli were the only glomeruli in the imaged region of the dorsal bulb that responded to nanomolar concentrations of $\beta$-phenylethylamine (Fig. 7B; Pacifico et al., 2012). Perhaps consistent with this high sensitivity, we also noted that T4 glomeruli were highly susceptible to adaptation. Even when stimulated at low concentrations, response amplitudes often decreased significantly with multiple odorant applications, making dose-response experiments particularly challenging. Despite this, it is clear that T4 glomeruli are highly sensitive and preferentially responsive to $\beta$-phenylethylamine.

Our patch-clamp recordings showed that T4-OSNs were more sensitive to the published T3 ligand cyclohexylamine than T3-OSNs. To directly compare the relative sensitivities of $\mathrm{T} 3$ and $\mathrm{T} 4$ in vivo, we recorded from mice in which both glomeruli were differentially labeled. Consistent with our in vitro data, cyclohexylamine activated both T3 and T4 glomeruli. Interestingly, the thresholds observed for both T3 and T4 in vivo were comparable (Fig. 7C,F), $\sim 500$ pM. The mean response amplitudes across all concentrations were not significantly different between $\mathrm{T} 3$ and T4 (ANOVA, $p=0.83$ ). Thus, T4 responds to both $\beta$-phenylethylamine and cyclohexylamine in vivo despite the fact that cyclohexylamine was excluded as a T4 ligand by previous studies (Liberles and Buck, 2006; Ferrero et al., 2012). In fact, T4 is at least as sensitive to cyclohexylamine as T3, although cyclohexylamine is one of the two most potent identified ligands for T3. Together, our
A
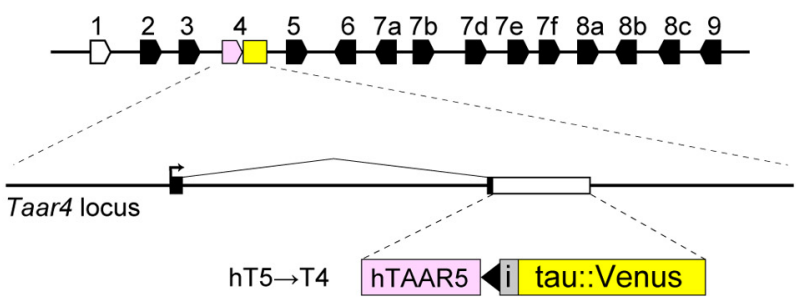

B
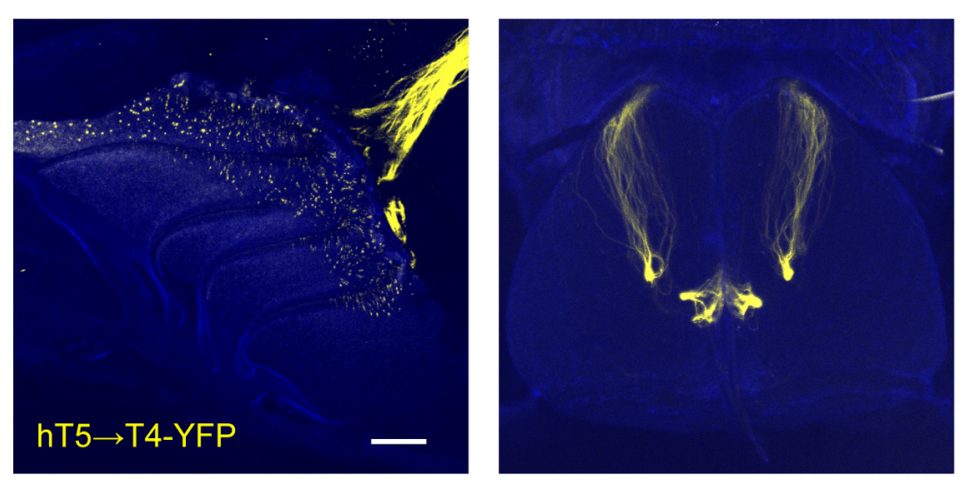

C T4-RFP

hT5 $\rightarrow$ T4-YFP
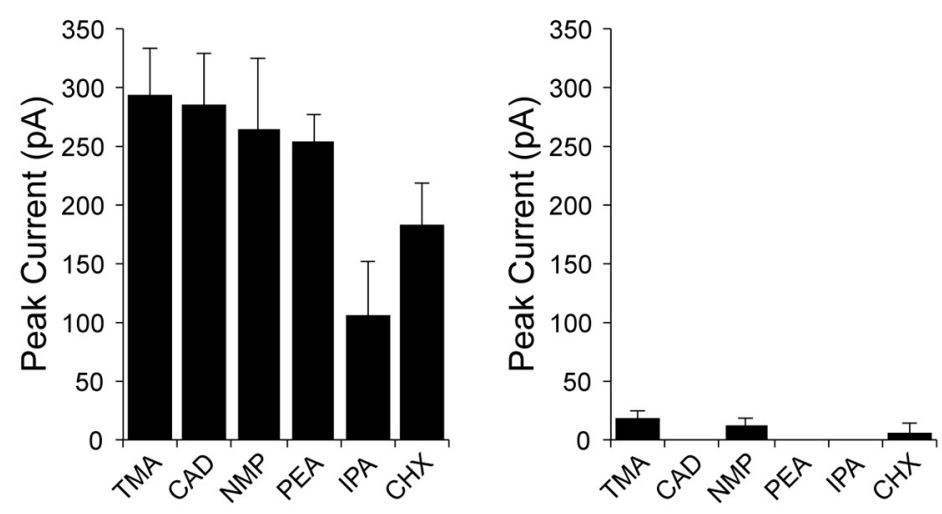

D
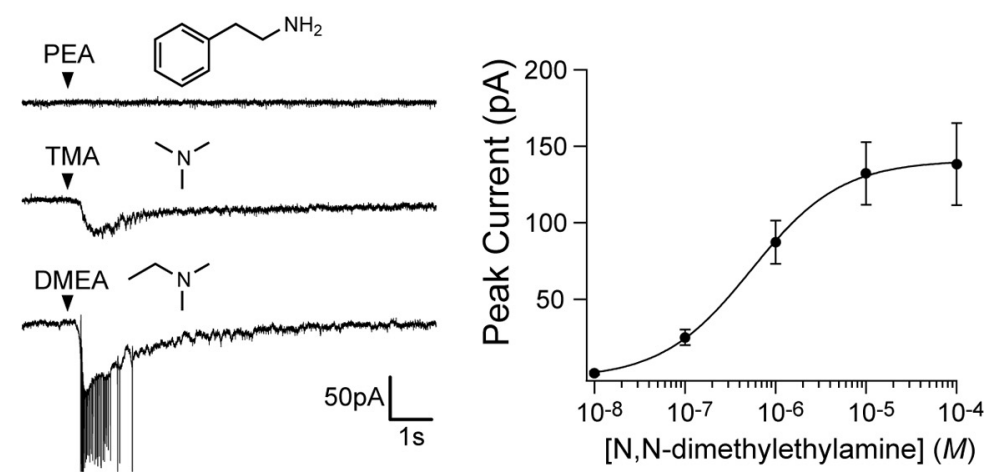

Figure 4. Amine responses are mediated by the expressed TAAR4 protein. $\boldsymbol{A}$, Diagram of the TAAR gene cluster and targeted allele. Polygons indicate gene orientation; olfactory TAARs are shown in black, and Taar1 is shown in white. The Taar4 locus is shown with coding sequence (white box), nontranslated regions (black boxes), and transcription start (arrow). The $h T 5 \rightarrow T 4$ targeted insertion swaps the mouse T4 coding sequence for that of hT5 (violet box), followed by an internal ribosome entry site (gray box marked "i") and the coding sequence for tauVenus (yellow box). LoxP site is shown (black triangle). $\boldsymbol{B}$, Left, Medial view of olfactory turbinates in an $h T 5 \rightarrow T 4$ homozygous mouse showingyellow-labeled OSN sand axons. Right, Dorsal view of the olfactory bulbs in an $h T 5 \rightarrow T 4$ heterozygous mouse showing location of axonal convergence to dorsal glomeruli. Scale bar, $500 \mu \mathrm{m}$. C, Average peak current elicited by odorants in T4-RFPOSNs ( $n=4$ cells) and hT5 $\rightarrow$ T4-0SNs ( $n=4$ cells) tested at $1 \mu \mathrm{m}$. Stimuli are trimethylamine (TMA), cadaverine (CAD), $\mathrm{N}$-methylpiperidine (NMP), $\beta$-phenylethylamine (PEA), isopentylamine (IPA), cyclohexylamine (CHX), acetophenone (ACP), and N,Ndimethylethylamine (DMEA). D, Current recordings from a single hT5 $\rightarrow$ T4 - OSN (left) and dose-response curve (right) for N,Ndimethylethylamine. Smooth line is least-squares fit of the Hill equation, $\mathrm{EC}_{50}=1.2 \pm 0.6 \mu \mathrm{m}$ ( $n=6$ cells). 
A
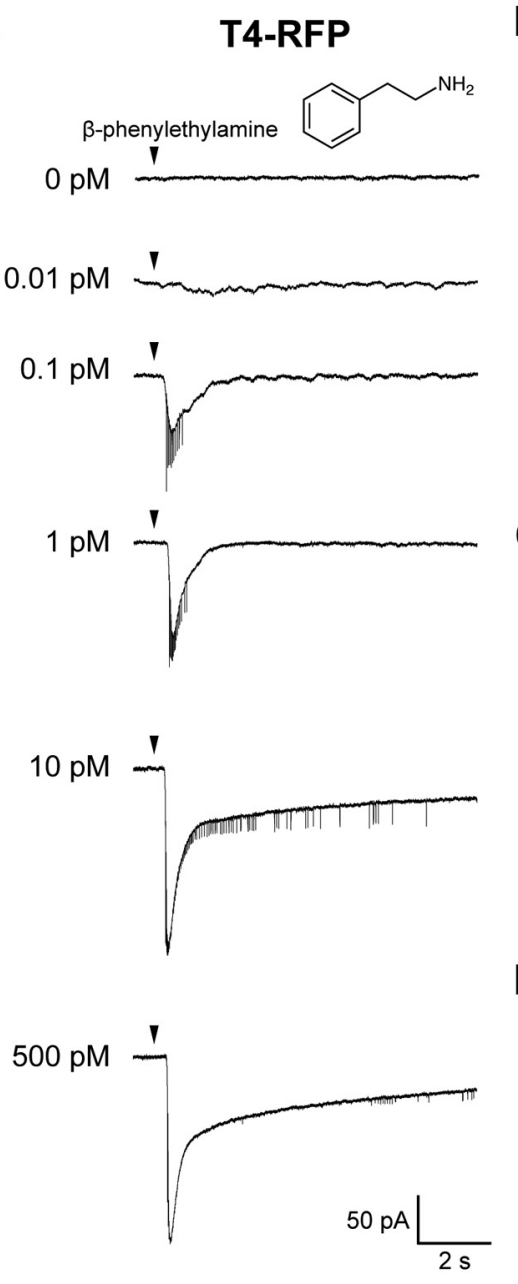

B
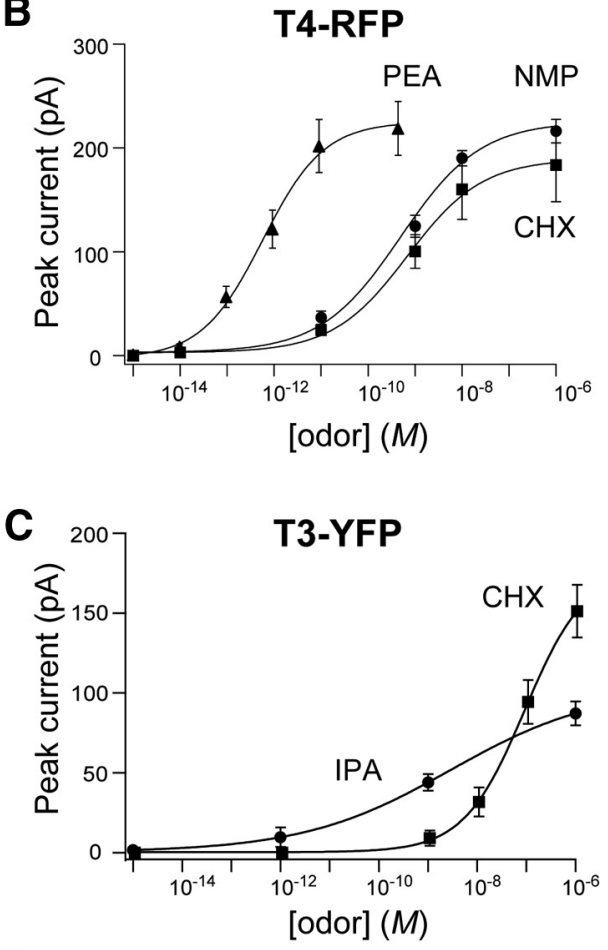

D

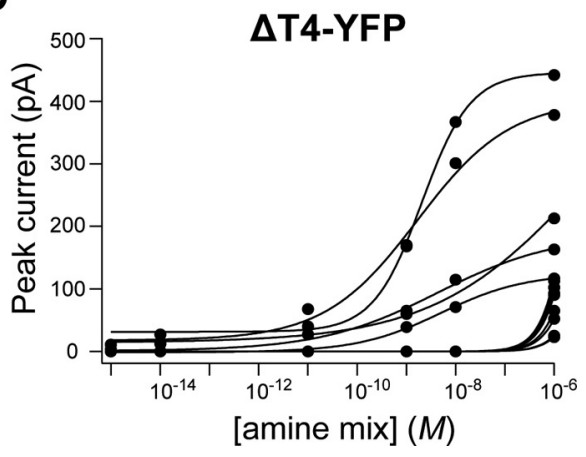

Figure 5. TAAR-expressing OSNs are highly sensitive to amines. A, Current recordings from a genetically identified T4-RFP OSN stimulated with $\beta$-phenylethylamine (PEA) at multiple concentrations (200 ms pulses). T4-0SNs responded with robust inward currents (downward deflections). B, Dose-response relationship of T4-RFP OSNs to $N$-methylpiperidine (NMP; $n=4$ cells), $\beta$-phenylethylamine (PEA; $n=8$ cells), and cyclohexylamine ( $\mathrm{CHX} ; n=4$ cells). $C$, Average dose-response data from T3-0SNs for isopentylamine (IPA; $n=4$ cells) and cyclohexylamine (CHX; $n=5$ cells). Additional data at higher concentrations were used to fit the Hill function, but the graph is cropped to match the range of $\boldsymbol{B}$ and $\boldsymbol{D}$ to allow direct comparison. $\boldsymbol{D}$, Individual dose-response relationship from $14 \Delta \mathrm{T} 4-0 \mathrm{SN}$ s from homozygous $\Delta \mathrm{T} 4-\mathrm{YFP}$ mice. OSNs were stimulated with multiple concentrations of an amines mix containing trimethylamine, cadaverine, $N$-methylpiperidine, $\beta$-phenylethylamine, propylamine, and cyclohexylamine. Because of adaptation in some cells, data are from the largest responses to each stimulus. Smooth lines are least-squares fits of the Hill equation.

data indicate that $\mathrm{T} 4$ has a relatively high sensitivity to amines in vivo as well as in vitro, even compared with other TAARs.

\section{Discussion}

Here we report, for the first time, the odorant response specificity of OSNs expressing genetically identified TAARs and their corresponding glomeruli in mice. We find that TAAR-expressing OSNs are selectively activated by amines, are broadly tuned at higher concentrations, and exhibit response thresholds that are several orders of magnitude lower than any previously published main olfactory chemoreceptor. This unprecedented sensitivity is most likely mediated by coupling to the canonical odorant transduction pathway. Our data provide insight into the unique contribution of the TAARs to vertebrate olfaction.

\section{Tuning breadth of the TAARs}

The response profiles observed in T3OSNs and T4-OSNs are notably different from those observed with heterologous expression of the same receptors (Liberles and Buck, 2006). We observe that the ligands identified for T3 and T4 in heterologous cells were the most effective stimuli for T3-OSNs and T4-OSNs. However, these OSNs also responded to structurally diverse amines, particularly at higher concentrations. Responses to these unexpected ligands are likely relevant for odor coding because they could be observed as increases in action potential firing rates in single OSNs and by glomerular activation in intact mice.

One potential explanation for the differences in tuning breadth observed using our in vivo expression approach is that the genetically tagged OSNs could express other receptors. However, the robust responses to all of the effective ligands were abolished when T4 was replaced with another receptor, indicating that the single, identified receptor mediates all of the observed odorant responses. Analyzing a receptor swap is preferable to analyzing a receptor deletion because OSNs that express a TAAR deletion allele go on to express other TAAR genes (Johnson et al., 2012; Pacifico et al., 2012). Nevertheless, we did analyze a population of homozygous $\Delta \mathrm{T} 4-\mathrm{OSN}$ and found that they rarely respond to cyclohexylamine and $N$-methylpiperidine, consistent with the idea that T4 mediates responses to these compounds as well (data not shown). Our observations argue strongly that the amine responses observed in our genetically tagged TAAR-OSNs are mediated directly by a single expressed receptor.

The most likely explanation for the difference between our data and previous results is that the TAARs exhibit different response profiles-or are simply more sensitive-when expressed in OSNs compared with cultured cells (Shirokova et al., 2005; Von Dannecker et al., 2005; Oka et al., 2006; Grosmaitre et al., 2009). OSNs

likely have specialized mechanisms for proper expression and localization of receptors (Matsunami et al., 2009). The readout of TAAR activation may be more sensitive in OSNs because the receptors are localized at high density in cilia and are coupled to the endogenous signal transduction cascade. Consequently, recording from the native cell type allows us to detect effective ligands that are missed in other assays. Our data support this view, because the response threshold for $\beta$-phenylethylamine in T4-OSNs was sub-picomolar: 1,000,000-fold lower than the previously reported T4 thresholds from cultured cells (Ferrero et al., 2011).

\section{Sensitivity of the TAARs}

In our experiments, we have measured the sensitivity of T3, T4, hT5, and a subset of randomly chosen mouse TAARs (from 
A
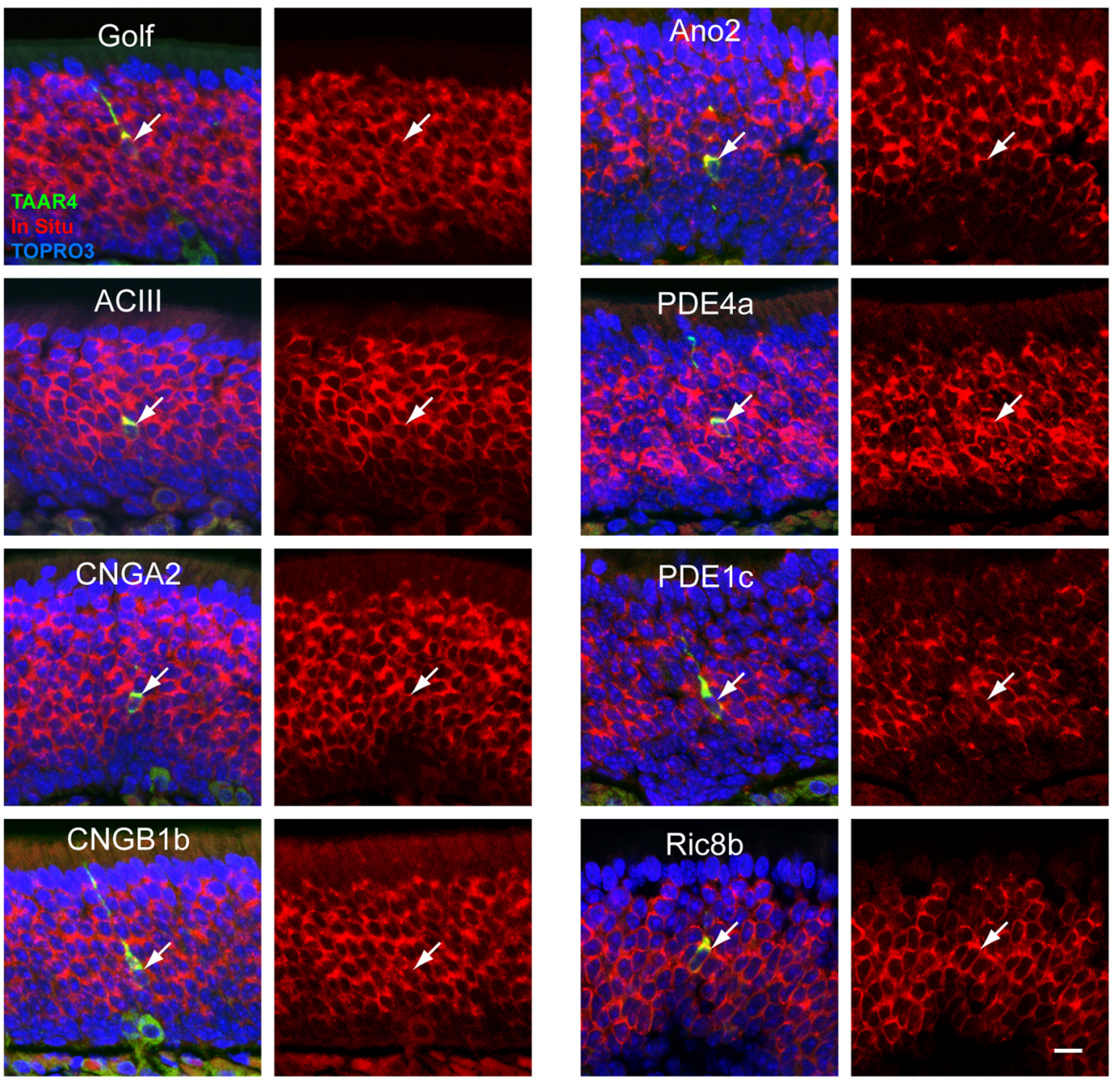

B

TAAR4

M72
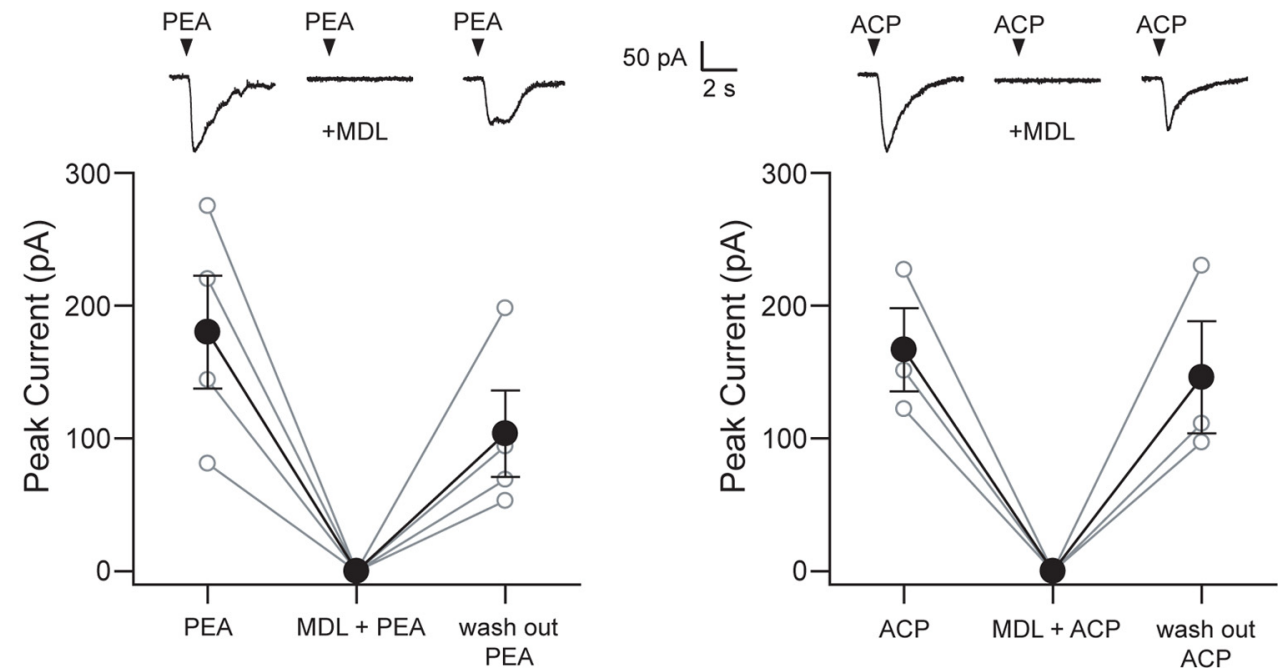

Figure 6. TAAR4-OSNs use the canonical odorant transduction cascade. A, Combined immunohistochemistry and fluorescence in situ hybridization for known olfactory signal transduction component genes in sections of the olfactory epithelium. T4-OSNs were visualized in T4-ChR-YFP mice (Pacifico et al., 2012) by staining with an antibody to GFP (green). In situ hybridization is shown for eight probes (red). Nuclei are stained with TOPRO-3 (blue). For each pair of panels, the left shows the combined channels, and the right shows the in situ signal only. Arrows mark locations of T4-OSNs. $\boldsymbol{B}$, Reversible block of odorant responses in T4-OSNs using the adenylyl cyclase blocker MDL-12,330A at $30 \mu \mathrm{m}$ (MDL). Response to $200 \mathrm{~ms}$ pulse of $1 \mathrm{~nm} \beta$-phenylethylamine (PEA; $n=$ 4 OSNs) was completely blocked and partly recovered after washout (left). A similar block was seen for OSNs expressing a canonical class II $0 R$ M72 ( $n=3$ ) stimulated with 200 ms pulses of $100 \mu \mathrm{M}$ acetophenone (ACP; right). Data for single cells are shown in gray and average data in black. Insets above show individual current traces. 


\section{A}
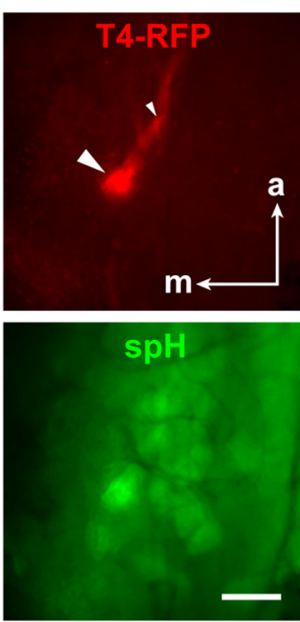

B
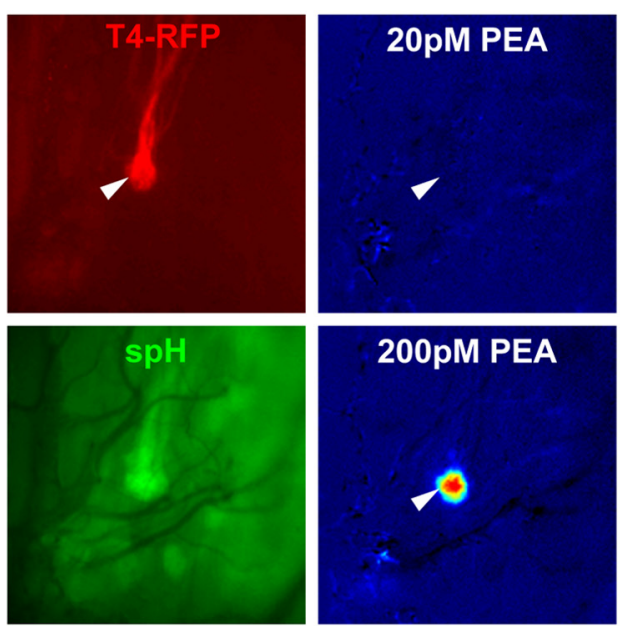

E
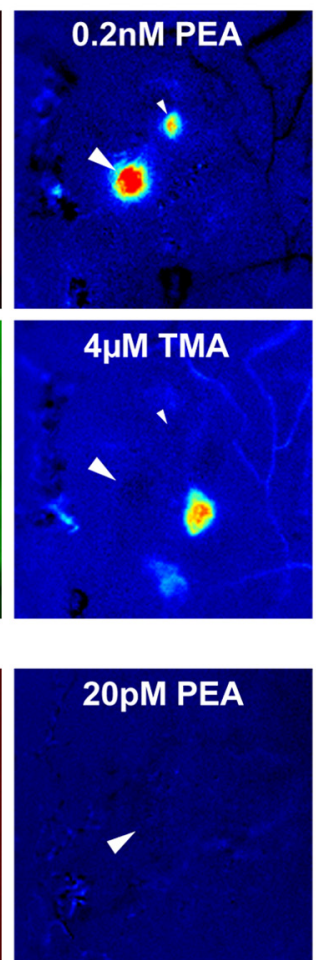

D

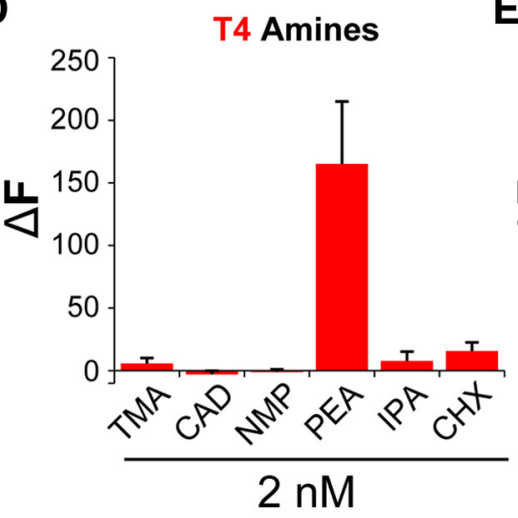

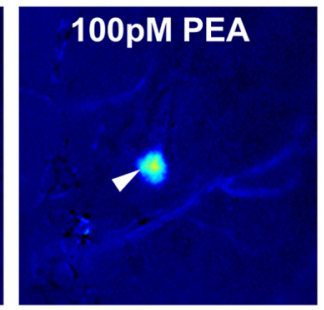
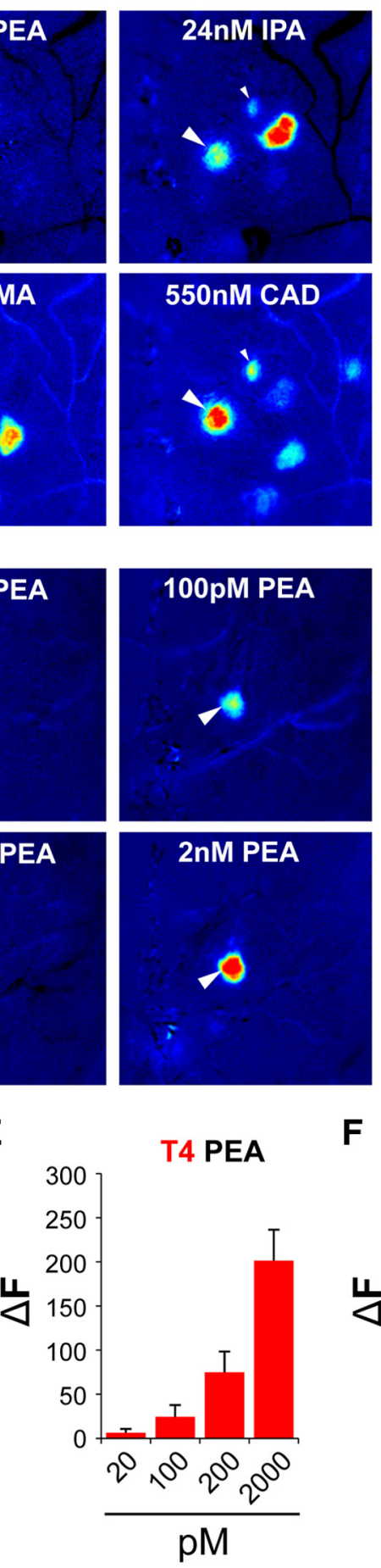
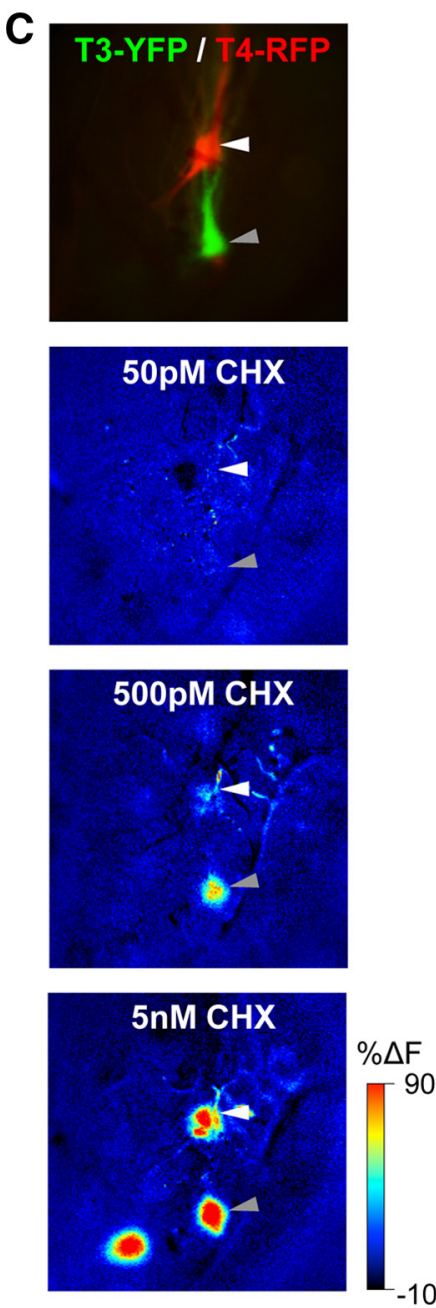

$\mathbf{F}$

$-10$

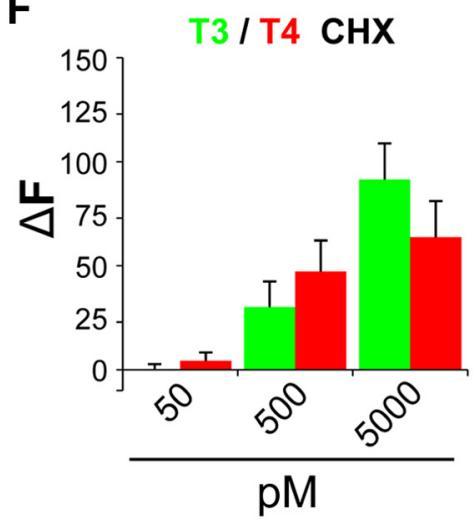

Figure 7. TAAR4 glomeruli are highly sensitive and broadly tuned in vivo. A, Optical imaging of odor-evoked spH signals from glomeruli in the dorsal, caudal olfactory bulb of a T4-RFP heterozygous mouse. Resting spH fluorescence (green panel) shows glomeruli, and RFP fluorescence (red panel) shows one large T4 glomerulus (large arrowhead) and one smaller T4 glomerulus (smaller arrowhead). Pseudocolored panels show responses to $\beta$-phenylethylamine (PEA), trimethylamine (TMA), and cadaverine (CAD) at the indicated vapor concentrations. $\boldsymbol{B}, 0$ ptical imaging of responses in T4 glomeruli of a heterozygous T4 -RFP mouse elicited with different concentrations of PEA. Resting spH fluorescence (green panel) shows glomeruli in the dorsal olfactory bulb. RFP fluorescence (red panel) shows a labeled T4 glomerulus (arrowhead). Pseudocolored panels show odor-evoked changes in fluorescence $(\Delta F)$ in response to increasing concentrations of $\beta$-phenylethylamine (PEA).C, Simultaneous recording from T3 and T4 glomeruli in the same mouse. Top shows resting fluorescence from T4-RFP (white arrowhead) and T3-YFP (gray arrowhead) glomeruli. Bottom three panels show responses to varying concentrations of cyclohexylamine (CHX). In this animal, T3 exhibited larger responses at threshold (500 pM). The highest $\mathrm{CHX}$ concentration elicited a large response in T3 and T4, as well as a more medial, neighboring glomerulus that most likely corresponds to another TAAR gene. D, Average response amplitude of T4 glomeruli to a set of amines at $2 \mathrm{~nm}$ vapor concentration ( $n=6$ glomeruli from 6 mice): $\beta$-phenylethylamine (PEA), cyclohexylamine (CHX), trimethylamine (TMA), cadaverine (CAD), and $N$-methylpiperidine (NMP). $\boldsymbol{E}$, Average response amplitude of T4 glomeruli to multiple concentrations of PEA ( $n=10$ glomeruli from 10 mice). $\boldsymbol{F}$, Average response amplitudes of T3 glomeruli (green) and T4 glomeruli (red). To improve sensitivity to $\mathrm{CHX}$, these recordings were made with the contralateral naris occluded, which increases flow rate and produces larger responses. Data are from five T3/T4 compound heterozygous mice, two T4-RFP mice, and one T3-YFP mouse. Data are mean \pm SEM. In A-C, anterior (a) is up, medial ( $m$ ) is to the left, and responses are scaled to the same maximum $\Delta F$. Scale bar, $200 \mu \mathrm{m}$. 
$\Delta \mathrm{T} 4-\mathrm{OSN}$ s) expressed in native OSNs. Of these, T4 was the most sensitive receptor. OSNs expressing canonical ORs typically respond to odorants in the micromolar range, depending on the functional assay used (Zhao et al., 1998; Kajiya et al., 2001; Bozza et al., 2002; Grosmaitre et al., 2006; Oka et al., 2006), although higher sensitivity has been reported in randomly chosen OSNs (Frings and Lindemann, 1990). Some of the most sensitive responses from genetically tagged OSNs have been observed by in vivo imaging of glomeruli expressing the OR " $\mathrm{mOR}-\mathrm{EG}$ " (Oka et al., 2006). mOR-EG glomeruli respond to eugenol in the nanomolar range, with thresholds $\sim 2 \times 10^{-8} \mathrm{M}$. In comparison, T4 glomeruli responded to $\beta$-phenylethylamine at a 200 -fold lower concentration in our imaging assay. Furthermore, the response threshold measured by electrophysiology was six orders of magnitude lower. Similarly, OSNs expressing the OR "SR1" respond with thresholds as low as $1 \times 10^{-12} \mathrm{M}$ using the same patchclamp assay used in our studies (Grosmaitre et al., 2009). In comparison, T4-OSNs are $\sim 100$-fold more sensitive. To our knowledge, T4-OSNs are the most sensitive, genetically identified OSNs yet described in the main olfactory pathway.

Indeed, the sensitivity of T4-OSNs rivals that of vomeronasal sensory neurons that respond to MHC peptides (Leinders-Zufall et al., 2004), which are the most sensitive mammalian chemosensory neurons yet examined. This is striking because vomeronasal neurons are more sensitive and more narrowly tuned than typical main olfactory OSNs (Leinders-Zufall et al., 2000), suggesting that the vomeronasal pathway may be specialized for detecting low concentrations. Our data suggest that the transduction pathways in the main olfactory and vomeronasal sensory neurons have a similar capacity to mediate responses from high-affinity receptors and indicate that at least some chemosensory receptors in the main olfactory pathway are similarly optimized to detect chemical cues at low concentrations.

Under our experimental conditions, T4-OSNs responded to stimulation with very few molecules of $\beta$-phenylethylamine. A concentration of $1 \times 10^{-14} \mathrm{M}$ represents $\sim 6000$ molecules per microliter. Our stimulation protocol results in ejection of $\sim 5.8 \mathrm{nl}$ of solution, or $\sim 35$ molecules of $\beta$-phenylethylamine (see Materials and Methods). At this concentration, the mean response amplitude was $\sim 8 \mathrm{pA}$. Assuming that $1 / 10$ of the molecules interact with the cilia, this gives $2.3 \mathrm{pA}$ per molecule, larger than the published unitary current responses in mouse OSNs (Ben-Chaim et al., 2011). However, differences in recording and stimulation methods make a direct comparison with previous studies difficult. In addition, unitary responses have never been characterized using TAARs or using ORs that have similarly high apparent affinities. Although our work suggests that T4-OSNs can detect very few molecules of $\beta$-phenylethylamine, additional analysis with more precisely controlled odorant delivery will be required to accurately determine the minimum number of molecules that can be detected by these neurons.

\section{Function of hTAAR 5}

Very little is known about the role of TAARs in human olfaction. Humans have six intact TAAR genes, including hTAAR1 (Lindemann et al., 2005), and expression of two (hTAAR5 and hTAAR8) have been reliably detected in human nasal mucosa biopsies (Carnicelli et al., 2010). However, it is unclear whether hTAARs encode fully functional receptors. Our data demonstrate directly that hT5 expression supports axon outgrowth and guidance to glomeruli when expressed in mouse OSNs- both of these functions are critically dependent on a functional receptor (Feinstein et al., 2004). In addition, we find that hT5 imparts a de- fined ligand specificity to mouse OSNs, providing the first direct evidence that hTAARs can function as chemosensory receptors.

Ligands have not been identified for human olfactory TAARs. We show that hT5 responds to the tertiary amine $\mathrm{N}, \mathrm{N}$ dimethylethylamine and to a lesser extent to trimethylamine, a structurally related agonist for mouse and rat TAAR5 (Liberles and Buck, 2006; Stäubert et al., 2010; Ferrero et al., 2012). Our data indicate that human and mouse $\mathrm{T} 5$ respond to structurally similar ligands, suggesting that they may be selected for (or have maintained) a common function. This is somewhat at odds with recent data showing that human and primate TAARs do not respond to the same odorants as their rodent orthologs (Stäubert et al., 2010). Additional characterization of the human and mouse TAARs in our assay should shed light on this issue.

Of the TAARs that we have studied, hT5 has the lowest sensitivity. The most likely explanation is that our limited screen did not identify high-potency ligands for hT5; our initial odorant set comprised only 40 amines. Alternately, it is possible that hT5 is generally less sensitive than the mouse TAARs. Additional experiments to define hT5 ligands will be required to distinguish between these possibilities. In any case, our data provide direct evidence that hTAARs are amine receptors and validate a novel approach to deorphanize the hTAAR repertoire via expression in the mouse.

\section{Function of the TAARs}

The TAARs likely serve a function in the main olfactory pathway that is not served by the much larger repertoire of canonical ORs. The data presented here support the assertion that the mouse TAARs have been selected as highly sensitive amine detectors (Pacifico et al., 2012). The functional significance of the unusual sensitivity of T4 reported here is not known. It could facilitate the detection of amines (such as $\beta$-phenylethylamine) that have relatively low vapor pressures and/or that may be present in the environment at low concentrations. Another (although not mutually exclusive) view is that T4 could mediate highsensitivity detection of behaviorally relevant compounds, such as kairomones, or social cues that are used for intra- or interspecific communication (Liberles and Buck, 2006; Ferrero et al., 2011) or generally aversive stimuli that might indicate the presence of toxins or unsafe food sources (Shalaby, 1996). Because $\beta$-phenylethylamine is a predator-derived odorant that can trigger aversive responses in rodents, it is tempting to speculate that the properties of T4 evolved (in rodents) to detect trace amounts of this signal in natural scent marks, a trait that would be of obvious adaptive value.

\section{References}

Ben-Chaim Y, Cheng MM, Yau KW (2011) Unitary response of mouse olfactory receptor neurons. Proc Natl Acad Sci US A 108:822-827. CrossRef Medline

Bozza T, Feinstein P, Zheng C, Mombaerts P (2002) Odorant receptor expression defines functional units in the mouse olfactory system. J Neurosci 22:3033-3043. Medline

Bozza T, McGann JP, Mombaerts P, Wachowiak M (2004) In vivo imaging of neuronal activity by targeted expression of a genetically encoded probe in the mouse. Neuron 42:9-21. CrossRef Medline

Bozza T, Vassalli A, Fuss S, Zhang JJ, Weiland B, Pacifico R, Feinstein P, Mombaerts P (2009) Mapping of class I and class II odorant receptors to glomerular domains by two distinct types of olfactory sensory neurons in the mouse. Neuron 61:220-233. CrossRef Medline

Bunting M, Bernstein KE, Greer JM, Capecchi MR, Thomas KR (1999) Targeting genes for self-excision in the germ line. Genes Dev 13:1524-1528. CrossRef Medline

Carnicelli V, Santoro A, Sellari-Franceschini S, Berrettini S, Zucchi R (2010) Expression of trace amine-associated receptors in human nasal mucosa. Chemosens Percept 3:99-107. CrossRef 
Feinstein P, Bozza T, Rodriguez I, Vassalli A, Mombaerts P (2004) Axon guidance of mouse olfactory sensory neurons by odorant receptors and the beta2 adrenergic receptor. Cell 117:833-846. CrossRef Medline

Ferrero DM, Lemon JK, Fluegge D, Pashkovski SL, Korzan WJ, Datta SR, Spehr M, Fendt M, Liberles SD (2011) Detection and avoidance of a carnivore odor by prey. Proc Natl Acad Sci U S A 108:11235-11240. CrossRef Medline

Ferrero DM, Wacker D, Roque MA, Baldwin MW, Stevens RC, Liberles SD (2012) Agonists for 13 trace amine-associated receptors provide insight into the molecular basis of odor selectivity. ACS Chem Biol 7:1184-1189. CrossRef Medline

Firestein S, Darrow B, Shepherd GM (1991) Activation of the sensory current in salamander olfactory receptor neurons depends on a $G$ proteinmediated cAMP second messenger system. Neuron 6:825-835. CrossRef Medline

Frings S, Lindemann B (1990) Single unit recording from olfactory cilia. Biophys J 57:1091-1094. CrossRef Medline

Grosmaitre X, Vassalli A, Mombaerts P, Shepherd GM, Ma M (2006) Odorant responses of olfactory sensory neurons expressing the odorant receptor MOR23: a patch clamp analysis in gene-targeted mice. Proc Natl Acad Sci U S A 103:1970-1975. CrossRef Medline

Grosmaitre X, Fuss SH, Lee AC, Adipietro KA, Matsunami H, Mombaerts P, Ma M (2009) SR1, a mouse odorant receptor with an unusually broad response profile. J Neurosci 29:14545-14552. CrossRef Medline

Hashiguchi Y, Nishida M (2007) Evolution of trace amine associated receptor (TAAR) gene family in vertebrates: lineage-specific expansions and degradations of a second class of vertebrate chemosensory receptors expressed in the olfactory epithelium. Mol Biol Evol 24:2099-2107. CrossRef Medline

Hussain A, Saraiva LR, Korsching SI (2009) Positive Darwinian selection and the birth of an olfactory receptor clade in teleosts. Proc Natl Acad Sci U S A 106:4313-4318. CrossRef Medline

Ishii T, Omura M, Mombaerts P (2004) Protocols for two- and three-color fluorescent RNA in situ hybridization of the main and accessory olfactory epithelia in mouse. J Neurocytol 33:657-669. CrossRef Medline

Johnson MA, Tsai L, Roy DS, Valenzuela DH, Mosley C, Magklara A, Lomvardas S, Liberles SD, Barnea G (2012) Neurons expressing trace amineassociated receptors project to discrete glomeruli and constitute an olfactory subsystem. Proc Natl Acad Sci U S A 109:13410-13415. CrossRef Medline

Kajiya K, Inaki K, Tanaka M, Haga T, Kataoka H, Touhara K (2001) Molecular bases of odor discrimination: reconstitution of olfactory receptors that recognize overlapping sets of odorants. J Neurosci 21:6018-6025. Medline

Kleene SJ (2008) The electrochemical basis of odor transduction in vertebrate olfactory cilia. Chem Senses 33:839-859. CrossRef Medline

Leinders-Zufall T, Lane AP, Puche AC, Ma W, Novotny MV, Shipley MT, Zufall F (2000) Ultrasensitive pheromone detection by mammalian vomeronasal neurons. Nature 405:792-796. CrossRef Medline

Leinders-Zufall T, Brennan P, Widmayer P, S PC, Maul-Pavicic A, Jäger M, Li $\mathrm{XH}$, Breer H, Zufall F, Boehm T (2004) MHC class I peptides as chemosensory signals in the vomeronasal organ. Science 306:1033-1037. CrossRef Medline
Liberles SD, Buck LB (2006) A second class of chemosensory receptors in the olfactory epithelium. Nature 442:645-650. CrossRef Medline

Lindemann L, Ebeling M, Kratochwil NA, Bunzow JR, Grandy DK, Hoener MC (2005) Trace amine-associated receptors form structurally and functionally distinct subfamilies of novel $\mathrm{G}$ protein-coupled receptors. Genomics 85:372-385. CrossRef Medline

Ma M, Chen WR, Shepherd GM (1999) Electrophysiological characterization of rat and mouse olfactory receptor neurons from an intact epithelial preparation. J Neurosci Methods 92:31-40. CrossRef Medline

Matsunami H, Mainland JD, Dey S (2009) Trafficking of mammalian chemosensory receptors by receptor-transporting proteins. Ann NY Acad Sci 1170:153-156. CrossRef Medline

McGann JP, Pírez N, Gainey MA, Muratore C, Elias AS, Wachowiak M (2005) Odorant representations are modulated by intra- but not interglomerular presynaptic inhibition of olfactory sensory neurons. Neuron 48:1039-1053. CrossRef Medline

Nagai T, Ibata K, Park ES, Kubota M, Mikoshiba K, Miyawaki A (2002) A variant of yellow fluorescent protein with fast and efficient maturation for cell-biological applications. Nat Biotech 20:87-90. CrossRef Medline

Nei M, Niimura Y, Nozawa M (2008) The evolution of animal chemosensory receptor gene repertoires: roles of chance and necessity. Nat Rev Genet 9:951-963. CrossRef Medline

Oka Y, Katada S, Omura M, Suwa M, Yoshihara Y, Touhara K (2006) Odorant receptor map in the mouse olfactory bulb: in vivo sensitivity and specificity of receptor-defined glomeruli. Neuron 52:857-869. CrossRef Medline

Pacifico R, Dewan A, Cawley D, Guo C, Bozza T (2012) An olfactory subsystem that mediates high-sensitivity detection of volatile amines. Cell Rep 2:76-88. CrossRef Medline

Shalaby AR (1996) Significance of biogenic amines to food safety and human health. Food Res Int 29:675-690. CrossRef

Shirokova E, Schmiedeberg K, Bedner P, Niessen H, Willecke K, Raguse JD, Meyerhof W, Krautwurst D (2005) Identification of specific ligands for orphan olfactory receptors. G protein-dependent agonism and antagonism of odorants. J Biol Chem 280:11807-11815. CrossRef Medline

Stäubert C, Böselt I, Bohnekamp J, Römpler H, Enard W, Schöneberg T (2010) Structural and functional evolution of the trace amine-associated receptors TAAR3, TAAR4 and TAAR5 in primates. PLoS One 5:e11133. CrossRef Medline

Stephan AB, Shum EY, Hirsh S, Cygnar KD, Reisert J, Zhao H (2009) ANO2 is the cilial calcium-activated chloride channel that may mediate olfactory amplification. Proc Natl Acad Sci U S A 106:11776-11781. CrossRef Medline

Von Dannecker LE, Mercadante AF, Malnic B (2005) Ric-8B, an olfactory putative GTP exchange factor, amplifies signal transduction through the olfactory-specific G-protein $\mathrm{G} \alpha_{\text {olf }}$ J Neurosci 25:3793-3800. CrossRef Medline

Zhang J, Huang G, Dewan A, Feinstein P, Bozza T (2012) Uncoupling stimulus specificity and glomerular position in the mouse olfactory system. Mol Cell Neurosci 51:79-88. CrossRef Medline

Zhao H, Ivic L, Otaki JM, Hashimoto M, Mikoshiba K, Firestein S (1998) Functional expression of a mammalian odorant receptor. Science 279:237-242. CrossRef Medline 